\title{
Closed-loop data-driven simulation
}

\author{
Ivan Markovsky* \\ School of Electronics and Computer Science \\ University of Southampton, SO17 1BJ, UK
}

\begin{abstract}
Closed-loop data-driven simulation refers to the problem of finding the set of all responses of a closed-loop system to a given reference signal directly from an input/output trajectory of the plant and a representation of the controller. Conditions under which the problem has a solution are given and an algorithm for computing the solution is presented. The problem formulation and its solution are in the spirit of the deterministic subspace identification algorithms, i.e., in the theoretical analysis of the method, the data is assumed exact (noise free). The results have applications in data-driven control, e.g., testing controller's performance directly from closed-loop data of the plant in feedback with possibly different controller.
\end{abstract}

Keywords: System identification; Subspace methods; Persistency of excitation; Data-driven simulation and control.

\section{Introduction}

The data-driven simulation problem is defined as follows: given a trajectory of an unknown system, find the response of that system to a different input signal (under specified initial conditions). Of course, data-driven simulation can be reduced to classical model-based simulation by identifying the unknown system from the given trajectory. In the case of linear time-invariant (LTI) systems, however, the problem can be solved without identifying a representation of the system in an intermediate step [MWRM05a, MWRM05b]. The resulting algorithm involves solution of a linear system of equations in which the left hand side is a Hankel matrix constructed from the given trajectory and the right hand side is the desired trajectory. Despite the computational simplicity of the basic idea, the theoretical analysis and computational details are not trivial due to the need to ensure that the given data is sufficiently informative, the need to organize the computations recursively and to deal with the initial conditions. Indeed, a data-driven simulation problem may not be solvable. The main assumptions for solvability are controllability of the system and persistency of excitation of the input that has generated the given trajectory.

The concept of data-driven simulation and the resulting computational algorithms have applications in system identification, e.g., computation of the impulse response from input/output data is a special data-driven simulation

\footnotetext{
*Email: imeecs.soton.ac.uk, Telephone: +44 (0) 238059 8715, Fax: +44 (0) 2380594498
} 
problem. Another application of data-driven simulation [MWVD06] is to give a system theoretic interpretation of the orthogonal and oblique projections, which appear in the subspace identification methods [VD96, VD92]. Finally, as shown in [MR07, MR08], data-driven simulation is the main building block of data-driven control methods, i.e., methods that derive the control signal directly from a trajectory of the plant without identifying a model of the plant.

This paper further develops the concept of data-driven simulation to closed-loop data-driven simulation, defined as follows: given an input/output trajectory of an unknown system and a representation of a controller, find all responses of that closed-loop system to a given reference signal. Our motivation for studying the closed-loop data-driven simulation problem comes from unfalsified control [ST97]. Unfalsified control is an switching adaptive control method that selects in real-time a controller satisfying the performance specification from a set of candidate controllers. The main step in unfalsified control is testing the performance of a candidate controller without applying it on the plant. The performance of the candidate controller is evaluated directly from data collected of the plant (possibly operating in closed-loop with another controller). Data-driven simulation allows us to evaluate the controller performance by computing the closed-loop behavior of the plant with the given controller. The standard performance test in the unfalsified control setting makes no assumptions about the plant (therefore it is applicable for a general nonlinear time-varying system), however, it computes a single trajectory of the closed-loop system, so that the performance test can be conservative. In contrast, closed-loop data-driven control uses an LTI assumption about the plant but computes the full behaviors of the closed-loop system, so that it is non-conservative in the LTI case.

A standing assumption throughout the paper is that the data is generated by an LTI system of bounded complexity. Admittedly, this assumption is practically unrealistic, however, it is convenient for the theoretical study ( $c f$. ., deterministic subspace identification) and trivial modifications of the algorithm-replace solution of a linear system of equation by an approximate solution, rank test by a numerical rank test, etc.-leads to practically useful algorithms that can cope with noise on the data ( $c f$., stochastic subspace identification). We envisage that stochastic version of the results presented in this paper will appear in near future.

\section{Notation}

We use the following standard notation: $\mathbb{R}$ is the set of real numbers, $\mathbb{N}$ is the set of natural numbers, and $\mathbb{R}^{\mathrm{w}}$ is the w-dimensional real vector space. $\left(\mathbb{R}^{\mathrm{w}}\right)^{\mathbb{N}}$ denotes the set of functions from $\mathbb{N}$ to $\mathbb{R}^{\mathrm{w}}$, i.e., $w \in\left(\mathbb{R}^{\mathrm{w}}\right)^{\mathbb{N}}$ is the time series

$$
w=(w(1), w(2), \ldots, w(t), \ldots), \quad \text { where } \quad w(t) \in \mathbb{R}^{w}
$$

$w \in\left(\mathbb{R}^{w}\right)^{T}$ is the finite sequence

$$
w=(w(1), w(2), \ldots, w(t), \ldots, w(T)), \quad \text { where } \quad w(t) \in \mathbb{R}^{\mathrm{w}},
$$

however, with some abuse of notation, we will view $w \in\left(\mathbb{R}^{w}\right)^{T}$ also as a wT-dimensional column vector. The concatenation of the finite sequence $w_{\mathrm{p}}$ with the (possibly infinite) sequence $w_{\mathrm{f}}$ is denoted by $\left(w_{\mathrm{p}}, w_{\mathrm{f}}\right) . A^{\dagger}$ is the pseudo-inverse of the matrix $A$ and $\operatorname{col} \operatorname{dim}(A)$ is the number of columns of $A$. 
The behavioural setting [Wil87] is especially suitable for solution of data-driven simulation and control problems because it treats a dynamical system as a set of trajectories (rather than equations) thus making explicit the relation between a trajectory and the system that generates the trajectory. In the behavioural setting, a discrete-time dynamical system $\mathscr{B}$ with w manifest variables (inputs and outputs) is a subset of the signal space $\left(\mathbb{R}^{w}\right)^{\mathbb{N}}$. In this paper, we assume that the manifest variables $w$ have a given input/output partition

$$
w=\left[\begin{array}{l}
u \\
y
\end{array}\right],
$$

where $u \in\left(\mathbb{R}^{\mathrm{m}}\right)^{\mathbb{N}}$ is an input and $y \in\left(\mathbb{R}^{\mathrm{p}}\right)^{\mathbb{N}}$ is an output. The restriction $\left.\mathscr{B}\right|_{T}$ of the behavior $\mathscr{B}$ to the interval $[1, T]$ is defined as

$$
\left.\mathscr{B}\right|_{T}:=\left\{w_{\mathrm{p}} \in\left(\mathbb{R}^{\mathrm{w}}\right)^{T} \mid \text { there is } w_{\mathrm{f}} \in\left(\mathbb{R}^{\mathrm{w}}\right)^{\mathbb{N}} \text { such that }\left(w_{\mathrm{p}}, w_{\mathrm{f}}\right) \in \mathscr{B}\right\},
$$

i.e., there is an extension $w_{\mathrm{f}}$ of a finite trajectory $\left.f_{\mathrm{p}} \in \mathscr{B}\right|_{T}$ of the system, to an infinite trajectory $w=\left(w_{\mathrm{p}}, w_{\mathrm{f}}\right) \in \mathscr{B}$.

The feedback interconnection of the plant $\mathscr{B} \subseteq\left(\mathbb{R}^{w}\right)^{\mathbb{N}}$ and a controller $\mathscr{C} \subseteq\left(\mathbb{R}^{\mathrm{r}+\mathrm{w}}\right)^{\mathbb{N}}$ is given by

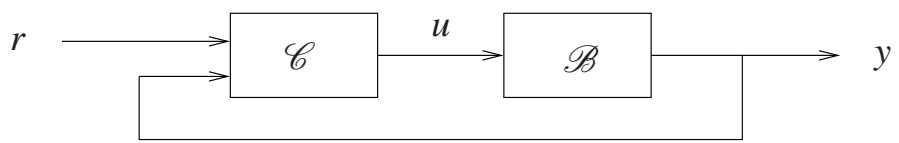

Figure 1: Feedback interconnection.

$$
\mathscr{B}_{\mathscr{C}}=\mathscr{B}_{\text {ext }} \cap \mathscr{C},
$$

where

$$
\mathscr{B}_{\mathrm{ext}}:=\left\{\left[\begin{array}{l}
r \\
w
\end{array}\right] \in\left(\mathbb{R}^{\mathrm{r}+\mathrm{w}}\right)^{\mathbb{N}} \mid w \in \mathscr{B}\right\} .
$$

We consider linear, time-invariant, and finite dimensional plants and controllers. A kernel representation $R(\sigma) w=0$, were $\sigma$ is the backwards shift operator

$$
\sigma w(t):=w(t+1)
$$

is parameterized by the polynomial matrix $R$, and an image representation $w=M(\sigma) g$ is parameterized by the polynomial matrix $M$.

The Hankel matrix with $t$ block rows, composed of the finite signal $w \in\left(\mathbb{R}^{\mathrm{w}}\right)^{T}$ is denoted by

$$
\mathscr{H}_{t}(w):=\left[\begin{array}{cccc}
w(1) & w(2) & \cdots & w(T-t+1) \\
w(2) & w(3) & \cdots & w(T-t+2) \\
w(3) & w(4) & \cdots & w(T-t+3) \\
\vdots & \vdots & & \vdots \\
w(t) & w(t+1) & \cdots & w(T)
\end{array}\right] .
$$


The signal $u=(u(1), \ldots, u(T))$ is called persistently exciting of order $L$ if the Hankel matrix $\mathscr{H}_{L}(u)$ is of full row rank. The banded upper-triangular Toeplitz matrix with $t$ block-columns, related to the polynomial

$$
r(z)=r_{0}+r_{1} z+\cdots+r_{n} z^{n}
$$

is denoted by

$$
\mathscr{T}_{t}(r):=\left[\begin{array}{ccccccc}
r_{0} & r_{1} & \cdots & r_{n} & 0 & \cdots & 0 \\
0 & r_{0} & r_{1} & \cdots & r_{n} & \ddots & \vdots \\
\vdots & \ddots & \ddots & \ddots & & \ddots & 0 \\
0 & \cdots & 0 & r_{0} & r_{1} & \cdots & r_{n}
\end{array}\right]
$$

\section{Closed-loop data-driven simulation}

Problem 1 (Closed-loop data-driven simulation). Given

- trajectory $w_{\mathrm{d}}=\left(w_{\mathrm{d}}(1), \ldots, w_{\mathrm{d}}(T)\right) \in\left(\mathbb{R}^{\mathrm{w}}\right)^{T}$ of a linear time-invariant system $\mathscr{B} \subset\left(\mathbb{R}^{\mathrm{w}}\right)^{\mathbb{N}}$, with an input/output partition $w=\left[\begin{array}{l}u \\ y\end{array}\right] \in \mathscr{B}, u \in\left(\mathbb{R}^{\mathrm{m}}\right)^{\mathbb{N}}$ input, $y \in\left(\mathbb{R}^{\mathrm{p}}\right)^{\mathbb{N}}$ output;

- linear time-invariant controller $\mathscr{C} \subset\left(\mathbb{R}^{\mathrm{r}+\mathrm{p}+\mathrm{m}}\right)^{\mathbb{N}}$, with an input/output partition $\left[\begin{array}{c}r \\ w\end{array}\right] \in \mathscr{C}, r \in\left(\mathbb{R}^{\mathrm{r}}\right)^{\mathbb{N}}, y \in\left(\mathbb{R}^{\mathrm{p}}\right)^{\mathbb{N}}$ inputs, $u \in\left(\mathbb{R}^{\mathrm{m}}\right)^{\mathbb{N}}$ output; and

- reference signal $r_{\mathrm{r}}=\left(r_{\mathrm{r}}(1), \ldots, r_{\mathrm{r}}\left(T_{\mathrm{r}}\right)\right) \in\left(\mathbb{R}^{\mathrm{r}}\right)^{T_{\mathrm{r}}}$

find the set of responses $w_{\mathrm{r}}$ of the closed-loop system $\mathscr{B}_{\mathscr{C}}$ to the reference signal $r_{\mathrm{r}}$.

\section{Solution and computational algorithm}

A closed-loop data-driven simulation algorithm aims to compute for given $w_{\mathrm{d}}, \mathscr{C}$, and $r_{\mathrm{r}}$, the signals $w_{\mathrm{r}}$, such that

$$
\left.\left[\begin{array}{l}
r_{\mathrm{r}} \\
w_{\mathrm{r}}
\end{array}\right] \in \mathscr{B}_{\mathscr{C}}\right|_{T_{\mathrm{r}}} \Longleftrightarrow\left\{\begin{array}{l}
\left.w_{\mathrm{r}} \in \mathscr{B}\right|_{T_{\mathrm{r}}} \\
{\left.\left[\begin{array}{l}
r_{\mathrm{r}} \\
w_{\mathrm{r}}
\end{array}\right] \in \mathscr{C}\right|_{T_{\mathrm{r}}}}
\end{array}\right.
$$

Assuming that the system $\mathscr{B}$ is controllable, it admits a minimal image representation

$$
\mathscr{B}=\left\{w=M(\sigma) l \mid l \in\left(\mathbb{R}^{\mathrm{m}}\right)^{\mathbb{N}}\right\} .
$$

Consider a minimal kernel representation of the controller

$$
\mathscr{C}=\left\{\left[\begin{array}{l}
r \\
w
\end{array}\right] \mid R_{r}(\sigma) r+R_{w}(\sigma) w=0\right\} .
$$

In terms of the image and kernel representations of the plant and controller, (4) becomes

$$
\left\{\begin{array}{l}
\text { there is } g, \text { such that } w_{\mathrm{r}}=M(\sigma) g \\
R_{r}(\sigma) r_{\mathrm{r}}+R_{w}(\sigma) w_{\mathrm{r}}=0 .
\end{array}\right.
$$


We can and do assume that the controller $\mathscr{C}$ is specified by a kernel representation, however, the plant $\mathscr{B}$ is only implicitly specified by the trajectory $w_{\mathrm{d}}$ and we aim to avoid using a representation of $\mathscr{B}$. The crucial step for doing this is to replace the image representation in (5) by the equation

$$
w_{\mathrm{r}}=\mathscr{H}_{T_{\mathrm{r}}}\left(w_{\mathrm{d}}\right) g .
$$

The vector $g$ is related to the input and initial conditions of the system that generate the trajectory $w_{\mathrm{r}}$. However, the mapping from $g$ to the system's input and initial conditions is not injective. (Note that a solution $g$ of (6) need not be unique.)

The equivalence of $w_{\mathrm{r}}=M(\sigma) g$ and (6) holds under the following assumptions

1. the system $\mathscr{B}$ is controllable,

2. the input component $u_{\mathrm{d}}$ of $w_{\mathrm{d}}$ is persistently exciting of order $T_{\mathrm{r}}$ plus the order of $\mathscr{B}$,

and is proved in [WRMM05, Theorem 1], see also [MWVD06, Section 8.4]. Therefore, under assumptions 1 and 2, the set of solutions $w_{\mathrm{r}}$ of the linear system of equations

$$
\begin{aligned}
& w_{\mathrm{r}}=\mathscr{H}_{T_{\mathrm{r}}}\left(w_{\mathrm{d}}\right) g \\
& \mathscr{T}_{\mathrm{T}_{\mathrm{r}}}\left(R_{w}\right) w_{\mathrm{r}}=-\mathscr{T}_{T_{\mathrm{r}}}\left(R_{r}\right) r_{\mathrm{r}} .
\end{aligned}
$$

is equal to the set of trajectories $w_{\mathrm{r}}$ solving the closed-loop data-driven simulation problem.

Note 2 (Multi-output systems). In (7), we have replaced the difference operator $R(\sigma)$ by the structured matrix $\mathscr{T}_{T_{\mathrm{r}}}(R)$. In the multi-output case, the structure of $\mathscr{T}$ is more complicated than the one shown in (3). In order to simplify the presentation and abstract from technical details, we assume that the system is single-output.

Substituting $w_{\mathrm{r}}=\mathscr{H}_{T_{\mathrm{r}}}\left(w_{\mathrm{d}}\right) g$ into the second equation of (7) gives the following system of equations

$$
\underbrace{\mathscr{T}_{T_{\mathrm{r}}}\left(R_{w}\right) \mathscr{H}_{T_{\mathrm{r}}}\left(w_{\mathrm{d}}\right)}_{A} g=\underbrace{-\mathscr{T}_{T_{\mathrm{r}}}\left(R_{r}\right) r_{\mathrm{r}}}_{b}
$$

The matrix $A$ is of dimension $T_{\mathrm{r}} \times(\mathrm{r}+\mathrm{w}) T_{\mathrm{r}}$, so that the system $A g=b$ is underdetermined. Let $g_{0}$ be a particular solution, e.g., the least-norm solution $g_{0}=A^{\dagger} b$ and let $N$ be a matrix whose columns span the null space of $A$. The set of solution of (7) for $g$ is

$$
\mathscr{G}:=\left\{g_{0}+N z \mid z \in \mathbb{R}^{\mathrm{coldim}(N)}\right\} .
$$

Then the set of responses $w_{\mathrm{r}}$ of the closed-loop system $\mathscr{B}_{\mathscr{C}}$ to the reference signal $r_{\mathrm{r}}$ is

$$
\mathscr{W}_{\mathrm{r}}=\mathscr{H}_{T_{\mathrm{r}}}\left(w_{\mathrm{d}}\right) \mathscr{G}=\{\underbrace{\mathscr{H}_{T_{\mathrm{r}}}\left(w_{\mathrm{d}}\right) g_{0}}_{w_{\mathrm{r}, 0}}+\mathscr{H}_{T_{\mathrm{r}}}\left(w_{\mathrm{d}}\right) N z \mid z \in \mathbb{R}^{\operatorname{coldim}(N)}\} .
$$

It is characterized by the particular response $w_{\mathrm{r}, 0}$ and a subspace- the column span of the matrix $\mathscr{H}_{T_{\mathrm{r}}}\left(w_{\mathrm{d}}\right) N$. Algorithm 1 summarizes the necessary steps for data-driven computation of $\mathscr{W}_{\mathrm{r}}$ from $w_{\mathrm{d}}, R$, and $r_{\mathrm{r}}$.

We proved that under the assumptions on the data $w_{\mathrm{d}}$ and the plant $\mathscr{B}$, specified in the derivation of the algorithm, Algorithm 1 solves Problem 1. 


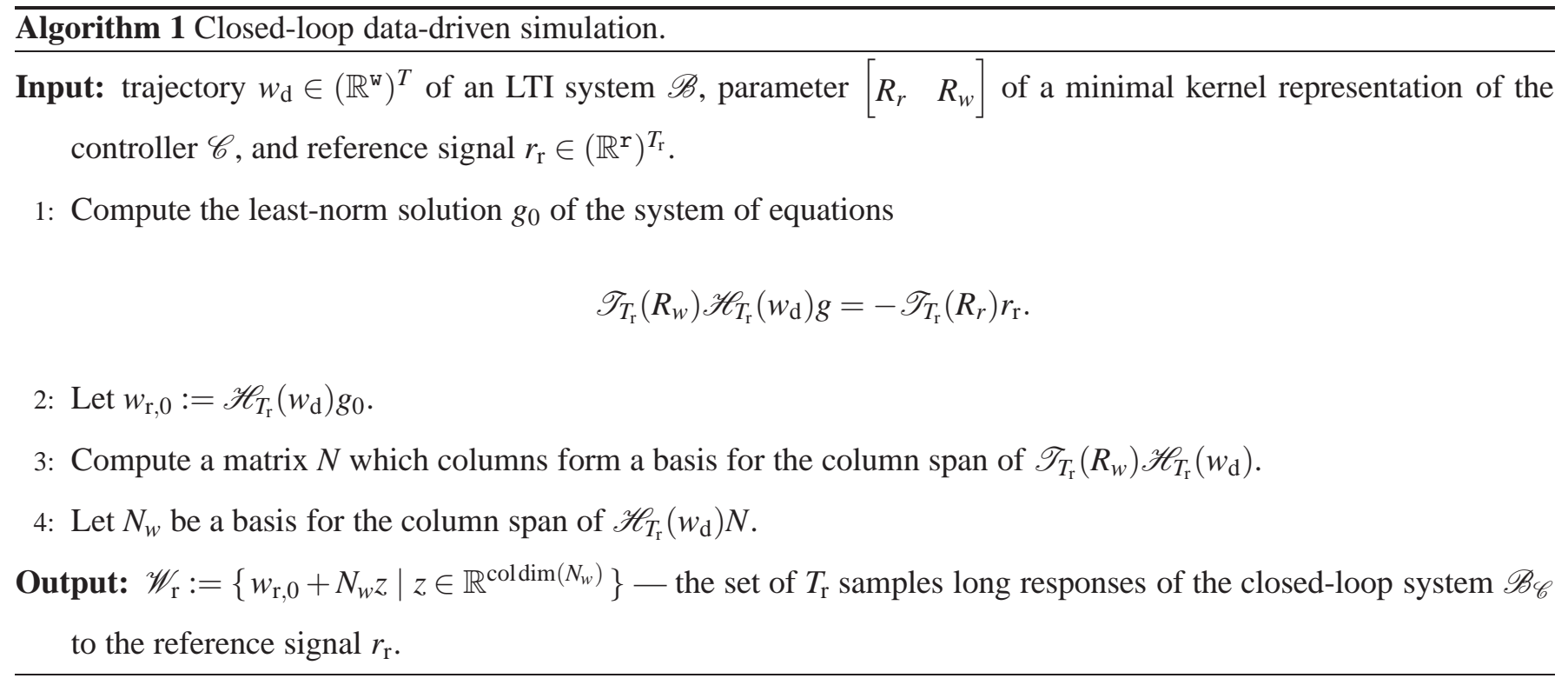

Theorem 3. Under the following assumptions:

1. the system $\mathscr{B}$ is controllable,

2. the input component $u_{\mathrm{d}}$ of $w_{\mathrm{d}}$ is persistently exciting of order $T_{\mathrm{r}}$ plus the order of $\mathscr{B}$,

the set

$$
\mathscr{W}_{\mathrm{r}}:=\left\{w_{\mathrm{r}, 0}+N_{w} z \mid z \in \mathbb{R}^{\mathrm{coldim}\left(N_{w}\right)}\right\},
$$

computed by Algorithm 1 is equal to the set of $T_{\mathrm{r}}$ samples long responses of the closed-loop system $\mathscr{B}_{\mathscr{C}}$ to the reference signal $r_{\mathrm{r}}$, i.e.,

$$
\mathscr{W}_{\mathrm{r}}=\left\{w \in\left(\mathbb{R}^{\mathrm{w}}\right)^{T_{\mathrm{r}}}\left|\left[\begin{array}{l}
r_{\mathrm{r}} \\
w
\end{array}\right] \in \mathscr{B}_{\mathscr{C}}\right|_{T_{\mathrm{r}}}\right\} .
$$

\section{Simulation example}

The data $w_{\mathrm{d}}=\left[\begin{array}{l}u_{\mathrm{d}} \\ y_{\mathrm{d}}\end{array}\right]$, used for the closed-loop data driven simulation, is the first 10 samples from the step response of a randomly generated first order system $\mathscr{B}$ interconnected with the controller $\mathscr{C}_{1}:=\left\{\left[\begin{array}{l}r \\ u \\ y\end{array}\right] \mid u=r-y\right\}$. The aim is to compute the first $T_{\mathrm{r}}=10$ samples of the step response of $\mathscr{B}_{\mathscr{C}_{2}}$, where $\mathscr{C}_{2}:=\left\{\left[\begin{array}{l}r \\ y \\ y\end{array}\right] \mid u=r+y\right\}$. For this purpose we use Algorithm 1, i.e., we do not compute explicitly a representation of $\mathscr{B}$. Note that either of the systems $\mathscr{B}_{\mathscr{C}_{1}}, \mathscr{B}_{\mathscr{C}_{2}}$ can be unstable.

In order to ensure that assumption 2 is satisfied, we augment the given trajectory—the step response of $\mathscr{B}_{\mathscr{C}_{1}}$ with $T_{\mathrm{r}}$ zeros. This takes into account the zero initial conditions of the given trajectory and ensures that the initial conditions of the computed response $r_{\mathrm{r}}$ of $\mathscr{B}_{\mathscr{C}_{1}}$ are also zero (i.e., $N_{w}=0$ ). The results for a particular system $\mathscr{B}$ are shown in Figure 2. We verify that up to numerical errors $r_{\mathrm{r}}$ matches the step response $s$ of $\mathscr{B}_{\mathscr{C}_{2}}$, obtained by model-based simulation. A Matlab file reproducing the simulation result is available from:

http://users.ecs.soton.ac.uk/im/test_cdds.m 

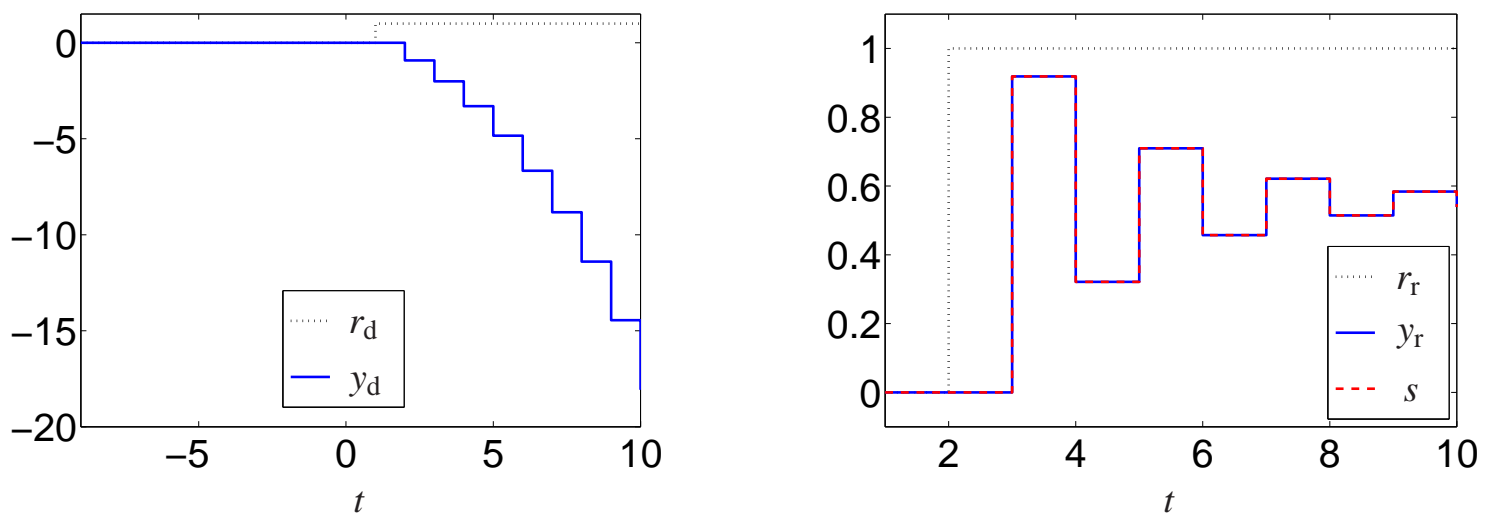

Figure 2: Step responses of $\mathscr{B}_{\mathscr{C}_{1}}$ (left) and $\mathscr{B}_{\mathscr{C}_{2}}$ (right).

\section{Application to data-driven control}

Data-driven control methods determine a control signal or a controller representation without using a model of the plant. The model is replaced by the observed data and prior hypothesis about the plant. Among the existing approaches for data-driven control, a particularly attractive one is the unfalsified control of Safonov and coworkers. As formulated in [ST97, SC01], unfalsified control uses no prior hypothesis on the plant apart from the observed data.

The unfalsified control is based on the observation that the ability of a candidate controller to meet desired performance specification can be tested using a trajectory of the plant without having a model of the plant or applying the controller on the plant. The controllers that (according to the test) fail to achieve the desired performance specification are discarded (falsified) and one of the remaining (unfalsified) controllers is used until it is falsified by the past measurements and replaced by a new unfalsified controller and so on. This leads to a switching adaptive control scheme. In this technical note, we do not consider a complete unfalsified switching adaptive control method but focus instead on its core ingredient- the test of a potential controller's performance directly from data of the closed-loop system, operating with a (possibly) different controller.

The standard test of the controller performance that uses only the data from the plant is based on what is called "fictitious reference" - a reference signal that together with the observed plant's output, could result (by proper choice of the controller's initial conditions) into an output of the controller that is equal to the observed plant's input. Therefore, according to the observed data of the plant, the tested controller driven by the fictitious reference (under suitable initial conditions) could have been in closed-loop with the plant. Consequently, the controller performance is verified against this closed-loop behavior. The controller is falsified on the basis of the observed data of the plant if the performance test (using the fictitious reference) fails. Otherwise, the controller is unfalsified by the data. The controller falsification procedure, based on a fictitious reference signal, is reviewed in Appendix A.

The fictitious reference test is conservative because (in the case of a static controller) a single trajectory of the closed-loop system is computed. Consequently, the performance of the controller is verified against this trajectory only. Using the LTI hypothesis, we can augment the computed closed-loop trajectory with all time shifts and sub- 
sequently with all linear combinations of the resulting set of trajectory. This construction is done systematically by the closed-loop data-driven simulation procedure. Moreover, according to Theorem 3, under assumptions 1 and 2, all trajectories of the closed-loop system are constructed in this way. Therefore, under these assumptions a test of the controller performance, based on the proposed closed-loop data-driven simulation procedure is non conservative.

\section{Conclusions}

We defined a new data-driven simulation problem, in which the closed-loop behavior of the unknown plant, in feedback with a given controller, is computed from a given trajectory of the plant and a representation of the controller. The proposed algorithm involves a solution of a linear system of equations and is, therefore, computationally fast and easy to implement. Future work will investigate modifications of the algorithm for recursive computation and noisy data, as well as application of closed-loop data-driven simulation in unfalsified and model predictive control.

\section{A Controller falsification based on fictitious reference}

In [ST97], the unfalsified control is defined ${ }^{1}$ for the feedback system of Figure 1. In a trajectory $w \in \mathscr{B}, w=:(u, y)$, $u \in\left(\mathbb{R}^{\mathrm{m}}\right)^{\mathbb{N}}$ is an input and $y \in\left(\mathbb{R}^{\mathrm{p}}\right)^{\mathbb{N}}$ is an output. Similarity, in $(r, u, y) \in \mathscr{C}, r \in\left(\mathbb{R}^{\mathrm{r}}\right)^{\mathbb{N}}$ and $y \in\left(\mathbb{R}^{\mathrm{p}}\right)^{\mathbb{N}}$ are inputs and $u \in\left(\mathbb{R}^{\mathrm{m}}\right)^{\mathbb{N}}$ is an output. The closed-loop system, obtained by interconnecting $\mathscr{B}$ and $\mathscr{C}$, is denoted $\mathscr{B}_{\mathscr{C}}$ and is given by (1).

The control specification can be formulated as a desired closed-loop behavior $\mathscr{B}_{\text {des }} \subseteq\left(\mathbb{R}^{\mathrm{r}+\mathrm{m}+\mathrm{p}}\right)^{\mathbb{N}}$. A controller $\mathscr{C}$ is said to achieve the desired behavior $\mathscr{B}_{\text {des }}$ (on the plant $\mathscr{B}$ ) if $\mathscr{B}_{\mathscr{C}} \subseteq \mathscr{B}_{\text {des }}$, i.e.,

$$
\mathscr{C} \text { achieve } \mathscr{B}_{\text {des }}(\text { on } \mathscr{B}) \quad: \Longleftrightarrow \quad \mathscr{B}_{\mathscr{C}} \subseteq \mathscr{B}_{\text {des }}
$$

Verification of (8) obviously requires knowledge of the plant's behavior $\mathscr{B}$. The aim of unfalsified control is to check if the controller fails to achieve the specification using only an observed trajectory $w_{\mathrm{d}}$ of $\mathscr{B}$.

Let $w_{\mathrm{d}}=\left(u_{\mathrm{d}}, y_{\mathrm{d}}\right) \in \mathscr{B}$ be a given trajectory of $\mathscr{B}$ and let $\mathscr{W}_{\mathrm{d} \text {,ext }}$ be the set of all possible extensions of $w_{\mathrm{d}}$ to a trajectory of $\mathscr{B}_{\text {ext }}$, i.e.,

$$
\mathscr{W}_{\mathrm{d}, \mathrm{ext}}:=\left\{\left(r, w_{\mathrm{d}}\right) \in \mathscr{B}_{\mathrm{ext}}\right\} .
$$

By construction $\mathscr{W}_{\text {d,ext }} \subset \mathscr{B}_{\text {ext }}$, so that $\mathscr{W}_{\text {d,ext }} \cap \mathscr{C} \subset \mathscr{B}_{\text {des }}$ is a necessary condition for $\mathscr{C}$ to achieve $\mathscr{B}_{\text {des }}$ (see (8)). It follows that a sufficient condition for $\mathscr{C}$ to fail to achieve $\mathscr{B}_{\text {des }}$ is

$$
\mathscr{W}_{\text {d,ext }} \cap \mathscr{C} \not \subset \mathscr{B}_{\text {des }} .
$$

The test (10) allows a controller to be falsified on the basis of the data alone without any assumption on the plant. Therefore, the test (10) is not only data-driven but also assumptions free.

\footnotetext{
${ }^{1}$ The presentation in [ST97] is restricted to the SISO case.
} 
The test (10) has a simple interpretation: $\mathscr{W}_{\mathrm{d} \text {,ext }} \cap \mathscr{C}$ is the problem of finding the set of reference signals $\mathscr{R}_{\mathscr{C}}\left(w_{\mathrm{d}}\right)$ that are consistent with the data $w_{\mathrm{d}}$ and the controller $\mathscr{C}$, i.e.,

$$
\mathscr{R}_{\mathscr{C}}\left(w_{\mathrm{d}}\right)=\left\{r \in\left(\mathbb{R}^{\mathrm{r}}\right)^{\mathbb{N}} \mid\left(r, w_{\mathrm{d}}\right) \in \mathscr{C}\right\}
$$

The signals $r \in \mathscr{R}_{\mathscr{C}}\left(w_{\mathrm{d}}\right)$ that satisfy (9) are called in [ST97] "fictitious reference signals". Of course, for given $\mathscr{C}$ and $w_{\mathrm{d}}, \mathscr{R}_{\mathscr{C}}\left(w_{\mathrm{d}}\right)$ may be an empty set (in which case the data $w_{\mathrm{d}}$ is not sufficient to falsify the controller $\mathscr{C}$ ). Computing $\mathscr{R}_{\mathscr{C}}\left(w_{\mathrm{d}}\right)$ for a general nonlinear controller $\mathscr{C}$ is a hard problem. For LTI controller, however, the problem is linear $-\mathscr{R}_{\mathscr{C}}\left(w_{\mathrm{d}}\right)$ is either an empty set or an affine space. Concrete algorithms for computing $\mathscr{R}_{\mathscr{C}}\left(w_{\mathrm{d}}\right)$ are given in [ST97]. Applied in real-time, these algorithms lead to an adaptive switching control strategy, see [WPSS05]. In this note, we do not consider the switching strategy and its real-time implementation but focus on its core ingredient- the fictitious reference test-in the case of an LTI plant.

\section{References}

[MR07] I. Markovsky and P. Rapisarda. On the linear quadratic data-driven control. In Proceedings of the European Control Conference, pages 5313-5318, Kos, Greece, 2007.

[MR08] I. Markovsky and P. Rapisarda. Data-driven simulation and control. Int. J. Control, 81(12):1946-1959, 2008.

[Mar09] I. Markovsky. An algorithm for closed-loop data-driven simulation. In 15th IFAC Symposium on System Identification, pages 114-115, Saint-Malo, France, 2009.

[MWRM05a] I. Markovsky, J. C. Willems, P. Rapisarda, and B. De Moor. Algorithms for deterministic balanced subspace identification. Automatica, 41(5):755-766, 2005.

[MWRM05b] I. Markovsky, J. C. Willems, P. Rapisarda, and B. De Moor. Data driven simulation with applications to system identification. In Proceedings of the 16th IFAC World Congress, Prague, Czech Republic, 2005.

[MWVD06] I. Markovsky, J. C. Willems, S. Van Huffel, and B. De Moor. Exact and Approximate Modeling of Linear Systems: A Behavioral Approach. Number 11 in Monographs on Mathematical Modeling and Computation. SIAM, March 2006.

[SC01] M. Safonov and F. Cabral. Fitting controllers to data. Control Lett., 43(4):299-308, 2001.

[ST97] M. Safonov and T. Tsao. The unfalsified control concept and learning. IEEE Trans. Automat. Control, 42(6):843-847, 1997. 
[VD92] M. Verhaegen and P. Dewilde. Subspace model identification, Part 1: The output-error state-space model identification class of algorithms. Int. J. Control, 56:1187-1210, 1992.

[VD96] P. Van Overschee and B. De Moor. Subspace Identification for Linear Systems: Theory, Implementation, Applications. Kluwer, Boston, 1996.

[Wil87] J. C. Willems. From time series to linear system-Part I. Finite dimensional linear time invariant systems, Part II. Exact modelling, Part III. Approximate modelling. Automatica, 22, 23:561-580, 675-694, 87-115, 1986, 1987.

[WPSS05] R. Wang, A. Paul, M. Stefanovic, and M. Safonov. Cost-detectability and stability of adaptive control systems. In Proc. IEEE Conf. on Decision and Control and European Control Conference, pages $12-15,2005$.

[WRMM05] J. C. Willems, P. Rapisarda, I. Markovsky, and B. De Moor. A note on persistency of excitation. Control Lett., 54(4):325-329, 2005. 